\title{
Phillips-type Approach or Acceleration?
}

IN THE CONTROVERSY OVBR MODBLS of inflation, I lean toward the accelerationist view. Two important recent contributions to the debate, by R. J. Gordon and George Perry, reported in this journal, have developed arguments on the other side by constructing Phillips-type models and by estimating their parameters from quarterly data for a period beginning in the early nineteen-fifties and ending in the late sixties. ${ }^{1}$ Both Gordon and Perry clearly feel that the evidence they have submitted weakens the accelerationist position. Perry, much more than Gordon, makes it clear that in the context of the Phillips debate he raises no claims beyond this. Indeed, some of the materials he presents will prove to be of considerable value quite aside from the Phillips controversy.

My doubts about the validity of the Phillips-type approach lead me to predict that policy makers will have a much better chance of success if they associate an accelerating rather than a constant rate of inflation with persistently maintained low rates of unemployment in the United States (such as a 4 percent rate, measured by the conventional American methods). However, a policy suggestion made in the final section of the paper will qualify this conclusion.

* Discussions with my colleague Henry C. Wallich were helpful, as always. I wish to thank Charles F. Stone for assistance in a more comprehensive project of which this paper is a by-product.

1. Robert J. Gordon, "The Recent Acceleration of Inflation and Its Lessons for the Future" (1:1970), pp. 8-41, and "Inflation in Recession and Recovery" (1:1971), pp. 105-58; and George L. Perry, "Changing Labor Markets and Inflation" (3:1970), pp. 411-41. 


\section{Defining the Phillips-type Approach from its Grass Roots}

The unit period for the data used in this section is a calendar year, which is not the case in the more elaborate models on which observations will be made in this note; compensation per manhour in the U.S. private nonfarm sector will be denoted by $w$, and the $\Delta w / w$ for period $t$ will stand for $\left(w_{t}-w_{t-1}\right) / w_{t-1}$; the "historical" acceleration in this series, $\Delta(\Delta w / w)$, will be measured for period $t$ by the difference between $\left(w_{t}-w_{t-1}\right) / w_{t-1}$ and $\left(w_{t-1}-w_{t-2}\right) / w_{t-2}$; analogous definitions will be used for the rate of increase and acceleration of the private nonfarm deflator $(P)$ and the consumer price index (CPI); and when such changes for period $t$ are related to the conventionally defined unemployment rate $(U)$, the rate in question will be $U_{t}$ measured in percent.

Statistically significant correlations are found for 1952-70 between the following pairs of variables, viewed separately:

(1) $\Delta w / w$ and $U$

(2) $\Delta(\Delta w / w)$ and $U$

(3) the rate of price increase ( $P$ or $C P I)$ and $U$

(4) the rate of price acceleration and $U$

(5) $\Delta U$ and $U$.

For (5), the regression coefficient is positive, while for (1) through (4) the coefficients are negative. The simple two-variable regressions on which these statements are based show zero acceleration of $w$ and also of $P$ and of $C P I$ when $U$ equals about 5 percent, and for this $U$ they show a rate of increase of $w$ of about 4.7 percent and a rate of price increase of about 2.2 percent.

By the Phillips-type approach, I mean one that introduces refinements into the relation described in (1) and (3) above, but, in a sense to be explained presently, denies analytical significance to the type of relation described in (2) and (4).

In the Phillips-type models the refinements of the relations described in (1) and (3) assume the form of the introduction of additional independent variables beyond $U,{ }^{2}$ and, in some cases, of the reconstruction of the unemployment variable by means of correcting the conventional $U$ by other

2. The variables explaining $\Delta P / P$ must, of course, include $\Delta w / w$. 
variables. When the parameters of the models are estimated, a negative relation is found between $\Delta w / w$ and also $\Delta P / P$ on the one hand, and the unemployment variable on the other. The coefficient, of course, changes its algebraic sign in the model whenever the reciprocal of unemployment or the employment rate is made to replace unemployment. Expected prices belong among the independent variables of the models under consideration, a fact that is simply noted here, though it may be added that a critical appraisal of the very simple assumptions about how this variable is generated from past price behavior will turn out to be the essential part of this paper. On these assumptions about expectations (to which I shall return), and on the assumption of given productivity trends at any given level of resource utilization (a reasonable simplifying assumption for the present purpose), there emerges the conclusion that for any given value of $U$, or of a reconstructed unemployment measure, $\Delta w / w$ and $\Delta P / P$ will remain unchanged, except for lags in the process of adjustment to some past change. This is nonacceleration in relation to the explanatory variables, none of which is assumed to change much in the long run if appropriately defined unemployment or labor market ease is held constant. Therefore correlations such as (2) and (4) are interpreted as having the misleading property of linking to $U$ itself a set of $\Delta(\Delta w / w)$ and $\Delta(\Delta P / P)$ values that have resulted from changes of $U$, or of a reconstructed unemployment measure. As was explained above, historically $\Delta U$ and $U$ are highly correlated, but the models imply that at any persistently maintained value of $U$, or of a reconstructed unemployment variable (labor market ease or tightness), the rate of inflation would have remained stable. This is the particular sense in which such models claim to be nonaccelerationist.

\section{The Question of Stability in Gordon's and Perry's Models}

The Gordon and Perry papers referred to suggest that on their basic behavioral assumptions neither writer would have found stability of their Phillips-type relations during their regression period as a whole had they not used a reconstructed measure of unemployment, or introduced variables that modify the effect of $U$ on inflation in view of some other measure of labor availability. The conventional $U$ did not decline sufficiently to explain the historically observed acceleration of inflation during the 
sixties; hence at that time acceleration did occur in relation to that $U$ (not merely historically). ${ }^{3}$

But if we make Gordon's corrections for changes in what he defines as disguised unemployment-that is, his numerical corrections for changes in the labor force participation rate and hence in the number of individuals who in some circumstances are and in others are not job seekers-and his similar corrections for the length of the workweek ("unemployment of hours"), then in the sixties Gordon's reconstructed unemployment measure declined appreciably more than did the conventional $U$. The Phillipstype function did show stability in relation to this corrected measure of unemployment or, with the algebraic sign reversed, in relation to the corresponding measure of the employment rate.

Perry's innovations are different from Gordon's and an attempt to use both sets of ideas for correcting $U$ would lead to shooting beyond the mark set by Phillips standards. Perry replaces the conventional $U$ with a weighted unemployment rate $\left(U^{*}\right)$ that takes account of the composition of the labor force and of the unemployed, thereby making allowances for the fact that the tightness-reducing effect of the availability of teenagers and of women is smaller than that of adult men, and of differential effects within these age-sex groups. The proportionate representation of adult men in the labor force was declining, particularly in the sixties, and the unemployment rates of that group as a whole have further declined in relation to

3. Even from the inevitably incomplete information about the bulky numerical materials the authors have used, it seems quite safe to infer this, and this section does so. The main part of the argument in the next section will not depend on this inference, though the reader will notice there too that $I$ have no doubt about its correctness. The final two sections imply nothing whatever in this regard.

Perry says enough about his adjustments, which will be briefly described in the text, to make the reader see that as the regression period progresses the adjustments lead to associating more and more predicted inflation with any given value of the conventional $U$, and that this effect is becoming particularly pronounced in the sixties, which from the accelerationist point of view is the "suspect" period. See Perry, "Changing Labor Markets," pp. 414, 417, 434.

Gordon's published materials do not contain enough about the details of his adjustments prior to 1964 to enable us to judge their effect for the early part of the regression period, and he has recently made major changes in the method. But he states clearly that for 1963-65 his reconstructed unemployment measure was unusually high in relation to the conventional $U$, and that this fact along with the sharpness of the subsequent reduction of his unemployment measure relative to the conventional $U$ "helps to explain why wage increases were so low in 1963-65 and so high in 1968-69." See Gordon, "Recent Acceleration of Inflation," pp. 16, 25; and "Inflation in Recession and Recovery," p. 133. 
the rates of the other categories. Since an unemployed teenager or woman enters into Perry's $U^{*}$ with a smaller weight than an adult man, $U^{*}$ was declining during the sixties more steeply than $U$, which helps to remedy the insufficiency of the decline of $U$ for a Phillips-type explanation of the historical acceleration of inflation observed in the sixties. Perry also suggests that "unemployment dispersion" (inequality of unemployment rates) among his age-sex groups is, other things equal, a significant inflationraising force. This dispersion has increased during the sixties, a fact that further helps him to explain the rising rate of inflation in the late years without assuming acceleration in relation to his specified variables. Moreover, Perry uses in his model $1 / U^{*}$ instead of $U^{*}$, as a result of which his measure shows a much greater absolute change when in an inflationary period unemployment is reduced from, say, 4.5 percent to 3.5 percent than when in a noninflationary period it is reduced from, say, 6 percent to 5 percent. This is because the absolute value of $(d / d x)\left(x^{-1}\right)=-x^{-2}$ rises with declining $x$.

The improvement achieved by introducing a variable reflecting the composition of the labor force can be seen even by using a variable $C$, defined as the percentage of the labor force consisting of males aged twenty and over. In this case the equation underlying the relation referred to as (1) above changes from

$$
\begin{aligned}
100(\Delta w / w)= & 9.01-0.86 U \\
& (8.44)(-3.97),
\end{aligned}
$$

with $R^{2}=0.51$, to the equation

$$
\begin{aligned}
100(\Delta w / w)= & 21.90-0.72 U-0.22 C \\
& (5.00)(-3.90)(-3.00),
\end{aligned}
$$

with $R^{2}=0.69$, and the standard error diminishes from 0.97 to 0.80 . At the same time the very large positive residuals for the inflationary years of the late sixties diminish appreciably: There is a substantial reduction of acceleration in relation to the independent variables when their number is increased merely from one to two in this particular fashion.

I feel convinced that Gordon's and Perry's innovations are based on sound general ideas. But this leaves wide open the question of whether the now available methods for making quantitative allowances for hidden unemployment and for the composition of the unemployment rate do not (perhaps inevitably) capture also policy-induced changes in the structural relation of expected to past values of variables. In the models this relation 
is assumed to have remained unchanged, yet if policy-induced inflationary changes did take place in that expectational relation, and if they were "corrected out" along with the allowances for hidden unemployment and for the composition of the unemployment rate, acceleration that should have been diagnosed was eliminated. This is because no significance (or even meaning) can be attached to a statement maintaining that historically rising values of $\Delta w / w$ and of $\Delta P / P$ have remained in the postulated relation to a set of independent variables-that is, have not accelerated in relation to these variables-provided that its validity requires corrections for inflationary changes of the postulated relations themselves as we move into the sixties.

The same proposition can be expressed more pointedly by the statement that methods such as those used in these models tend to eliminate any acceleration that may be present in the data and hence are not suitable for discovering whether the data do or do not point to acceleration. The basic reason for this is the absence of any trustworthy independent criteria for deciding whether the quantitative allowances that lead to good results in a Phillips-type model are more appropriate than those that do not accomplish this objective. To take the position that those ways of making the allowances that do lead to stable Phillips tradeoffs are the superior ones would, of course, mean prejudging the issue.

How plausible are the adjustments that are incorporated into the models? Gordon first assigns the same weight to his disguised unemployment (a rapidly declining variable during the inflationary years of the sixties) as to the conventional $U$ (a very mildly declining variable). In his later article he replaces this procedure by estimating separate coefficients for specific components of his unemployment concept but he runs into difficulties as a result of correlation between the components, and he specifies his unemploymenttype variables as disguised unemployment and unemployment of hours and unemployment dispersion (Perry's concept) instead of the first two and the conventional $U$; also, in his later article he changes his estimate of disguised unemployment, and the decline of his revised measure from the mid-sixties to the end of the decade becomes even more pronounced. ${ }^{4}$ All this seems forced and not very plausible.

Perry's reconstruction of the unemployment variable-ease-of-labor-

4. See Gordon, "Inflation in Recession and Recovery," p. 133, along with the coefficient of $U^{D}$ in equation (11), p. 118, and compare this with the difference between columns 1 and 2 in Table 2, "Recent Acceleration of Inflation," p. 21. 
market variable-I do not regard as "forced" or "implausible," but while the two contributions differ substantially in this regard, it cannot be maintained that by some independent criterion Perry's reconstruction is superior to allowances that would lead to poor results in a Phillips-type framework (nor does Perry present his findings in a spirit of "overclaiming"). For example, as he correctly points out, his weighting of the age-sex groups would in itself take care of the composition problem if the groups were perfect substitutes for one another. Since they are not, one would like to have a measure indicating how the substitution weights become altered when substitution (change of input proportions) takes place. Such a measure is not available, and, indeed, Perry tells us that for his very broadly defined groups he finds in his data no appreciable changes of weights during the regression period. Hence he supplements the use of constant substitution weights with a measure (unemployment dispersion) that provides some information about the likelihood that substitution will in fact have to take place when resource utilization increases. Such substitution presumably should have the consequence that the weights will change, though not only do we not know by how much but we must even admit that no changes are observable in the data. Perry does obtain good results for his regression period, and does so with reliance on an expedient for which, in view of the great difficulties faced, a case can indeed be made; yet the argument for using this particular expedient, in addition to using $1 / U^{*}$ instead of $U^{*}$, has no unique plausibility when the procedure is compared with those that would fail to keep the Phillips tradeoffs stable.

Given the present state of information, we do not know whether the policy changes from the fifties to the sixties did or did not change the relation between expected and past price movements during Gordon's and Perry's regression period, thus producing acceleration in the relevant sense. Hence it would be wrong to assert that such structural changes have in fact been concealed by the extent of upward correction of the rate of decline of the unemployment variable in the models under consideration. But, in the absence of dependable criteria for correcting the conventional $U$, the methods that appear to be most suitable for making the corrections are at the same time methods most suitable for suppressing whatever acceleration may be present in the data.

The next section briefly discusses the implications of the opposing views on whether, for their regression period, Gordon's and Perry's models have eliminated acceleration that should have been spotted. About this, doubts 
will be expressed rather than a firm answer suggested. The following section presents much firmer views along these same lines about the inherent lack of suitability of Phillips-type models for predicting the future and, in particular, about the seriousness of the mistake involved in judging the consequences of new policy departures by the results obtained from these models. This is an essential point because important new policy departures are often advocated with reference to the "stability" of models belonging in this class.

\section{Expectations-generating Structures and their Changes}

Gordon, Perry, and most other authors derive the public's expectations of future price increases, an important variable in the models, from price increases of the past. Among other measures of price expectations, Gordon includes in one place in his later analysis (without much emphasis) also a measure based on an informal opinion survey, which cannot, however, be depended on for reasonably precise information about the variable in question. At any rate, if we wanted to treat expectations as exogenous, the distinction between the stability and instability of our models would essentially disappear. Nonacceleration of inflation in relation to exogenous inflationary expectations that may well accelerate would be a useless concept.

By "expectations-generating structure" I mean the relation between expected magnitudes and observed past magnitudes that an investigator uses for deriving the expectations he attributes to the public. This belongs among the basic relations describing a Phillips-type model, relations assumed to remain unchanged during the period to which the data apply. However, the past magnitudes that are thus made to "explain" the expected ones inevitably form merely a very small part of the experience on which individuals base their expectations, since in practice much of that experience is incapable even of being identified. Further, the effects on expectations of much of the identifiable part of the experience are extremely complex. In the event of major changes in the public's appraisal of the behavior of policy makers, it is exceedingly unlikely that the expectations-generating structure used in formal analysis would not change. If in spite of such changes we observe "good behavior" in a model based on an immutable expectations-generating structure, we have reason to suspect that some 
other variable has performed not only its rightful function but also that of covering up a structural change, a service that it will hardly continue to perform in future periods. The statement applies to all models from which expectations structures are estimated, including various interest rate models (for example, those used by Gordon). ${ }^{5}$

The following analysis justifies the view that major changes in the expectations-generating structure "should" have taken place during Gordon's and Perry's regression period, although we cannot be certain about the length of the lag with which the public's reaction was in fact forthcoming.

Leaving aside the atypical opening, "Korean," years of the fifties, there were during the 1954-63 interval four occasions on which $\Delta w / w$ exceeded 0.04 (the time unit remains the calendar year); on only two of the four occasions did $\triangle P / P$ and $\triangle C P I / C P I$ rise into the range between 0.03 and 0.04; on three of the four occasions the policy line adopted was such that $U$ rose promptly from the year in question to the next, and on one occasion it remained for a year at a level as high as 5.5 percent and rose in the following year. During these sequences of events $U$ twice rose into the range between 6.5 and 7 percent. Alternations of expansionary policies

5. In contrast with Perry, Gordon uses interest rate equations for estimating the most appropriate lag structure of the past price increases that are supposed to determine the public's price expectations. The expectations-generating structure so estimated is then carried over into a Phillips-type model in which price expectations belong among the explanatory variables (in addition to the reconstructed unemployment variable and some others).

The interest rate equations express a hypothesis concerning the determination of real rates of interest and they explain the difference between money and real rates by price expectations. Not only are the underlying interest theories inevitably very far-reaching oversimplifications but I find it impossible even to interpret the interest theory sketched by Gordon in "Recent Acceleration of Inflation," pp. 36-37, in any logically acceptable way (the monetary authority is said to set the ratio of money to income and thereby to influence interest rates "at least temporarily"). Gordon revised the hypothesis in "Inflation in Recession and Recovery," pp. 146-47. Here the demand for real output, as a determinant of real rates, seems to be represented by what in the Keynesian framework is a multiplicand; yet since during the inflationary span of the sixties-in contrast with earlier periods-the multiplier applicable to this multiplicand diminished significantly, in that span the multiplicand used by Gordon rises too fast to represent the demand for output; and since therefore during that span a correct measure of demand would have risen at a lesser rate than Gordon's (that is, would have shown up with a lesser "real-rate raising effect"), here too the question is left open whether the expectations structure (explaining the difference between money and real rates) has not changed in the inflationary direction. 
and of restraints resulted in large fluctuations of $U$, between 4.1 and 6.8 percent. The outcome of the 1960 elections suggested policy changes but these did not occur promptly.

In these regards a new era started after 1963, during which $U$ was 5.7 percent, a figure that by 1969 declined to 3.5 percent. For 1963-64, $\Delta w / w$ was 0.048 , with a decline of $U$ to 5.2 percent by 1964 , followed promptly by a further decline of $U$ to 4.5 percent in 1965. Subsequently, while $U$ was declining toward its 1969 value of 3.5 percent, $\Delta w / w$ was rising well beyond $0.07, \triangle C P I / C P I$ well beyond 0.05 , and $\triangle P / P$ well beyond 0.04 . It is true that from the middle of 1968 on various types of restraint were applied - first mainly of a fiscal rather than a monetary nature and then the other way around-but these did not have much effect until 1969 or even 1970. During the recession year 1970 policy clearly shifted to the stimulation of a recovery; for that year $\Delta w / w$ was $0.066 ; \Delta C P I / C P I, 0.059 ; \Delta P / P, 0.048$; and $U, 4.9$ percent (though the highest seasonally corrected monthly $U$ was 6.2 percent).

Thus, it is reasonable to say that, in the first part of the regression period, rational expectations "should" have borne a relation to preceding price developments different from that in the second part. A "recent" $\Delta w / w$ of 0.045 should give rise to very different expectations concerning next year's $\Delta w / w$ depending on whether the policy makers are or are not believed to take a view of 0.045 similar to that the city traffic police take of 75 miles an hour. Only accidentally could this difference be captured by a long-drawnout lag structure within a given relation between expected and past price changes. To those years of the sixties in which the inflationary process was gathering momentum the analogy with the traffic police clearly does not apply. The government was known to be increasing its expenditures rapidly; it took several years before the public could have felt that tax increases might be around the corner; and with one dramatic but short interruption the Federal Reserve was increasing the money supply rapidly, in part at least in an unsuccessful effort to prevent interest rates from rising to high levels. If the expectations-generating structure did react with reasonable promptness to changes in the policy line, then, as was said above, a Phillips-type system can have given the appearance of stability only because the model builders made some overadjustments for the shortcomings of the conventional unemployment measure. However, it is conceivable that the public reacted very slowly. The fact that no firm judgment can be expressed about this is perhaps a matter of secondary importance, because 
even if the expectations structure reacted merely with a very long lag, the carry-over from the sixties, as well as future changes in the public's appraisal of policy making, will necessarily place the predictive power of Phillips-type systems in jeopardy.

Gordon has dealt with the problems that did in fact call for model revisions during the recession year $1970 ;{ }^{6}$ but being concerned more with longer-run predictive power in the event of policy changes, the analysis here now turns to the presumptive consequences of building future policy on the allegedly stable inflation-unemployment tradeoff that can be inferred from Phillips-type models.

\section{Dangers of Basing Policy on Estimates from Phillips-type Systems}

The dangers this section discusses result from the near-certainty that at "low" levels of $U$ the policies based on Phillips-type systems would keep changing the expectations-generating structure and that they would lead to accelerating inflation. As will be explained presently, a "low" level of $U$ is to be interpreted in this context as one at which excess demand for specific types of labor becomes a phenomenon of appreciable importance. ${ }^{7}$ There is reason to assume that, given our methods of measuring unemployment, 4 percent is such a level of $U$ at the present stage of American economic development, except for the qualification to be added in the last section of this paper.

Why is inflation more pronounced when $U$ is persistently maintained at 4 percent than when it is maintained at a higher level? The reason is that the labor force consists of a very large number of categories, distinguished by a large variety of criteria, and by the time the aggregate $U$ has declined to what by present standards is a "low" level, shortages develop in many of these specific categories. Many of these shortages are "physical," though a good many others are "man-made" in the sense that they result from barriers to entry (which policy makers should, of course, try hard to eliminate). The available physical equipment, too, is heterogeneous, and as a result of bottlenecks inflation can start even at high levels of $U$. But if such

6. Gordon, "Inflation in Recession and Recovery."

7. The reasons for this excess demand over available supply at given money-wage rates include institutional supply restrictions, and the excess demand develops in view of the expected monetary and fiscal policies. 
inflation is not short-lived and if plant and equipment of various sorts can be produced without much delay, $U$ is likely to be reduced to a level at which shortages develop also in many of the distinguishable labor categories. The fact that $\Delta w / w$ usually increases to some extent also for the other labor categories reflects in part the institutional characteristics of the wage structure of enterprises. These characteristics usually "come through" even with respect to nonunionized labor if the excess supply in the more amply available categories is small. The whole structure is moved up by specific shortages that develop in different countries at different levels of $U$, depending on the relationship between demand for and supply of specific labor categories.

The very great likelihood of acceleration in these circumstances derives from the inability of decision-making units to achieve their objectives by the moves they make during any period, and from a strong presumption that in a permissive environment the size of the moves will therefore keep growing. What follows in this section is an elaboration on this proposition. Alternatively (if the reader wishes to use terminology some implications of which I find unconvincing), what follows now is an elaboration on the proposition that, given the postulated specific shortages, the unemployment level here considered falls short of the "natural" level.

When $U$ is held at a level at which the excess demand for the specific labor categories in question is greater than was the case at an earlier level, $\Delta w / w$ also will be greater, but as a result of price increases the general trend in real wage rates will not become steeper. ${ }^{8}$ Insofar as importance attaches to the efforts of unions to obtain higher real wages when the national labor market becomes tighter, this difference in effort obviously becomes a source of acceleration. Yet Arthur Okun is right in reminding us that such a statement does not in itself settle the problem because, given the competitive bidding of employers for labor, the individual workers accepting the money-wage offers they find most favorable thereby accept also the most favorable real-wage offers, and not all economists would agree that unions are consistently trying for higher real wage rates when $U$ is 4 percent than they are when it is 5 percent. The question, therefore, is whether acceleration should be expected to originate also in the bidding of employers for individual workers in the scarce categories.

8. This is true unless long-run productivity trends should be steeper at the "low" level of $U$ that is under consideration here than they are at the higher level with which the comparison is made. 
Given the postulated policy commitment to maintain macro-economic labor scarcities at a chosen level, the answer is in the affirmative. In each successive round of bidding the employers who prove to be the gainers of scarce labor are those who, led by correct judgment, bid high enough to achieve this objective, and the losers of scarce labor will be those who made the incorrect judgment of not bidding high enough or of following suit merely for a reduced number of workers. In these circumstances the bidding must be expected to become increasingly sharp and inflation to accelerate. Given the postulated policy commitment, we are not entitled to the assumption that rising money wage rates will relieve the scarcities by creating a need for the reallocation of labor among sectors and hence will lead to a matching of the excess labor demand of some employers by a release of labor on the part of others (who then would have proved right in not keeping up with the gainers). We are not entitled to this assumption because the postulated policy is expansionary enough not to allow the tightness of the labor market to become reduced at the rising level of money wage rates, regardless of reallocations.

A policy building on the conception of stable Phillips tradeoffs cannot help but be permissive in the sense here implied. Consider the problems that would arise if policy makers, guided by Phillips-model estimates, decided to stabilize $U$ at 4 percent in the belief that this corresponds to a continuous sequence of $\Delta w / w=0.07$ and $\Delta P / P=0.045$ without acceleration. ${ }^{9}$ These would be estimates derived from data of a period in which policy makers did not act in this fashion, and in which the expectations structure was influenced by the experience that $U$ has increased whenever $\Delta w / w$ or $\Delta P / P$ rose beyond some range. As a result of the pressures discussed above, the private sector would in these circumstances almost certainly "test" the policy makers to see whether they persist in the policy of keeping $U$ at 4 percent even if (in contrast to Phillips-inspired beliefs) acceleration does develop, or in that event will use restraints for rolling back $\Delta w / w, \Delta P / P$, and $\triangle C P I / C P I$.

In the former case - that is, permissive policy-the expectations-generating structure would become very different from what it was in the period whose data were used for making the Phillips estimates; disappointed expectations would result in continued changes of the structure and in con-

9. Roughly speaking, this belief would be consistent with Perry's results; see, "Changing Labor Markets and Inflation," p. 432. 
tinued acceleration as long as the policy makers persisted in holding $U$ at 4 percent. In the latter case- the application of restraints because of acceleration at a $U$ of 4 percent-the policy would cease to be Phillips-based. It would become a policy trying to prevent $\triangle P / P$ from becoming greater than 0.045 by allowing $U$ to fluctuate to the extent required for achieving this end. Furthermore, if we have in mind the United States and take at their face value the figures here used for illustration (as nonaccelerationists might), it is appropriate to add that a policy designed to keep $\triangle P / P$ at 0.045 would be exposed to more obstinate "testing" by the public and hence would probably have to resort to harsher restraints than a policy that first succeeds in reducing price inflation into the range of, say, 2 percent to 3 percent: This is true because an American policy trying from now on for a $\Delta P / P$ of 0.045 would be interpreted as having revised its conception of normalcy under pressure, and it would take time and great consistency to convince the public that further upward revisions will not follow. The stop-and-go policies needed for preventing "historical" acceleration would in these circumstances become very onerous, particularly because the United States does not belong in the group of countries for which the stop phases of such policies mainly involve a reduced rate of resource transfer from the agricultural to the industrial sector, or reduced admission of foreign workers. Nor does the United States belong among the countries in which discrepancies between the composition of demand and that of supply in the labor market would start becoming disturbing only at exceedingly low levels of $U$.

\section{Are the Implied Hardships Necessarily Severe?}

The prevention of accelerating inflation and the achievement of a reasonably stable growth path with high productivity increases are very likely to require a higher rate of unemployment, as measured by the usual American methods, than has come to be considered the desirable target rate. Recognition of this fact would somewhat increase the small fraction of the labor force experiencing unemployment of extended duration and the individuals added to this group would be bona fide job seekers capable of doing useful work. The important question that arises is whether systematic arrangements could be made-not simply emergency measures taken in a recession-to secure for these persons work opportunities in the public sector. 
To serve the present purpose, the method of financing would, of course, have to be noninflationary, but, in principle at least, there is no reason why inflationary methods would have to be used. Concerning the risk that such a program would create or accentuate resource scarcities a reasonable degree of optimism may be expressed, because-unless something goes wrong with the administration of such a program - the individuals in question would not fall into one of the many categories in which shortages tend to develop but into a category in which there is excess supply. Reduction of this excess supply would not in itself create shortages.

Yet it is undeniable that answers would have to be found to questions of great complexity, such as the question of work morale. A fruitful solution would involve rendering services in the public sector on terms that are satisfactory and yet do not tempt exodus from conventional jobs and thus do not create severe problems of enforcement for the administering agency.

These are difficult questions and they are very important ones, deserving thorough exploration. There should at any rate exist less costly ways of taking care of the interests of a small proportion of the labor force than that of throwing the entire economy into a high degree of inflationary instability with the probable consequence that the rate of productivity increase becomes reduced across the board. One would hope for better ways also than that of further enlarging the welfare rolls, in this case with persons honestly interested in work opportunities; and for better ways than changing open inflation into suppressed inflation by means of a comprehensive system of controls. These, it seems to me, are the only alternatives to the suggestion made in the present section. Phillips-curve optimismthe theory of the stable tradeoff-has detracted attention from these problems. 